\title{
FURTHER OBSERVATIONS ON COUPLED EXTRASYSTOLES AND AUTOMATIC VENTRICULAR RHYTHMS
}

\author{
BY
}

\author{
A. SCHOTT AND D. SCHERF \\ From the Cardiographic Department of Queen Mary's Hospital for the East End, London, England, and the Department \\ of Medicine, New York Medical College, New York, N.Y., U.S.A.* \\ Received May 13, 1958
}

A few years ago we reported three cases of atrial fibrillation with idioventricular automatic beats, in which extrasystoles with fairly accurate coupling followed only automatic ventricular beats, but never conducted supraventricular ones (Scherf and Schott, 1951). We pointed out that such observations represent a contribution to the relation between ectopic beats with accurate coupling to the preceding, initiating, beat, and those with varying coupling which we consider due to a different, namely, automatic origin.

A tentative explanation was offered that this unusual arrhythmia may be due to a directional protective block of the ectopic centre, resulting from refractory tissue in the vicinity of the centre, which permits the initiating impulse to reach the ectopic focus only from certain directions. It was emphasized that analysis of more tracings of this rare disturbance of rhythm would be needed before more definite conclusions about the underlying mechanism could be drawn.

The present report, based on three new observations showing the same phenomenon in association with other features, is offered as a further contribution to gain a better understanding of this rare arrhythmia, whose underlying mechanism is still far from clear.

\section{CASE REPORTS}

Case 1. The patient was a 51-year-old woman, suffering from syphilitic aortic regurgitation and hypertension, the blood pressure being 205/100. The cardiogram showed left ventricular hypertrophy and a digitalis effect. Throughout her stay in hospital the patient received $0 \cdot 2 \mathrm{~g}$. of digitalis folia a day.

Fig. 1 shows that the dominant rhythm was sinus rhythm, rate 88-94 a minute (cycle length 64-68) $\dagger$. The ventricular complexes of the sinus beats have high $\mathbf{R}$ waves with slight slurring of the ascending limb, small s waves, distorted S-T segments, and diphasic T waves. An arrhythmia is present due to ventricular ectopic beats occurring with couplings varying between 39 and 62 in the reproduced portion (36-62 in the whole record). The ectopic beats have small r, deep S, and upright $\mathrm{T}$ waves; their shape can best be recognized in those beats where they are least distorted by fusion with sinus beats (beats marked by $\mathrm{x}$ in which the initial ventricular deflections are undistorted, but the final deflections are modified by superimposed $\mathbf{P}$ waves of the following sinus beat). Numerous fusion beats are present. These features strongly suggest parasystole, and measurement of long strips confirms this diagnosis, showing the inter-ectopic intervals to vary between the extremes of 108 and 116, or multiples thereof. Pressure on the right carotid sinus suppressed the atrioventricular conduction, the heart action being exclusively dominated by the ectopic ventricular focus throughout carotid sinus pressure (except for one beat, Fig. 1, d, see below). The shape of these beats is identical with the undistorted shape of the ectopic ventricular beats during parasystole. The first ectopic cycle measured 108, subsequently it gradually increased to 116 and a maximum of 124 (between the 9th and 10th beat), then gradually fell to 116 toward the end of the period of entirely ectopic rhythm which altogether comprised 42 cycles. After the 20th beat, a conducted beat, possibly an atrial extrasystole, occurred with a coupling of 100 (Fig. 1,d); the following cycle measured 124, that is, equalled the idioventricular cycle length prevailing at the time. These time relations resemble those observed with ventricular extrasystoles in complete A-V block and suggest interference with the impulse formation in the centre of the dominant rhythm.

* Aided by grants from M. Intrator and H. Petschek.

$\dagger$ All figures indicating intervals in cardiograms are expressed in hundredths of a second. 
a

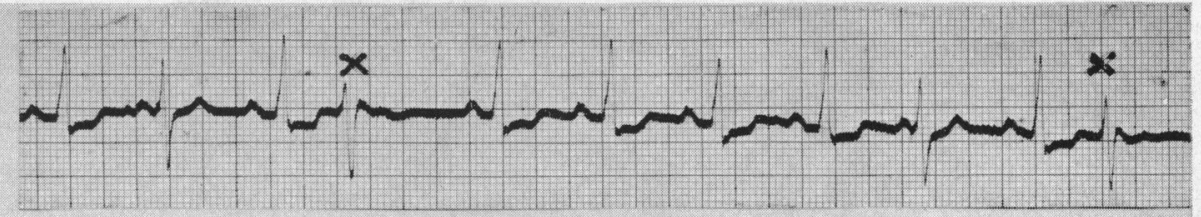

b

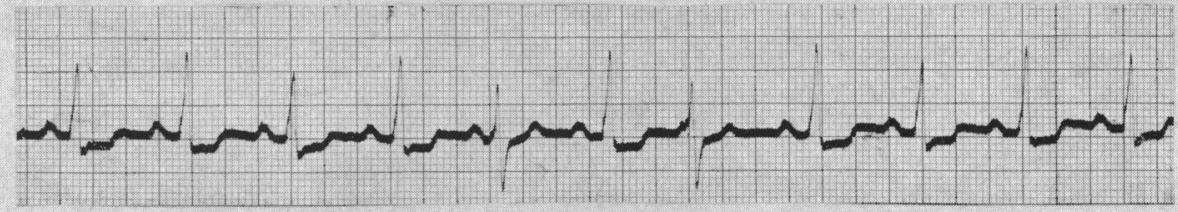

C

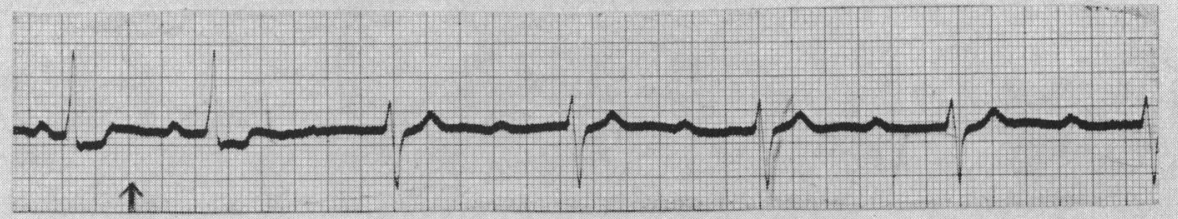

d
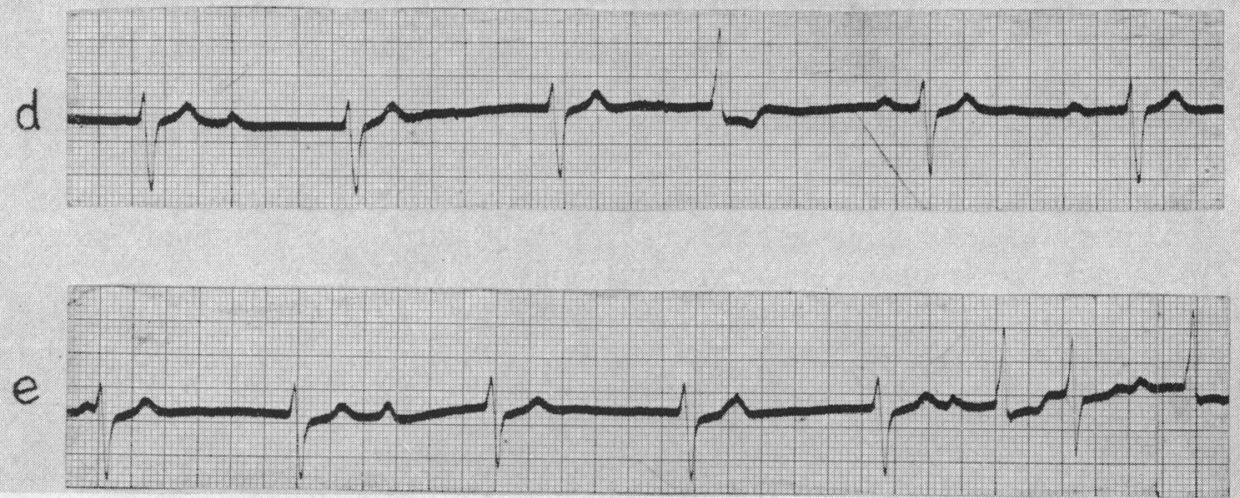

FIG. 1.-Case 1, lead II. Strips a-c are continuous, a and b show parasystole. At arrow in $\mathrm{c}$ beginning of right carotid sinus pressure, resulting in suppression of $\mathrm{A}-\mathrm{V}$ conduction; ventricular action solely due to parasystolic centre. First ectopic cycle length 108, last 116. Strip d: 18th to 22nd cycle of ectopic rhythm, carotid sinus pressure continuing. Cycle length fairly constant at 124 . The interval after the isolated conducted beat (in the middle of the strip) had the same length. Strip e: starting at the end of the 42nd cycle during carotid sinus pressure and immediately after its release.

Fig. 1, c and d, reproduces representative portions of the record obtained during carotid sinus pressure. When pressure on the sinus was released, the original arrhythmia became re-established (Fig. 1, e). This effect was reproducible: when the right carotid sinus was compressed for a second time, the same result on the arrhythmia was obtained; the period of ectopic rhythm during carotid sinus pressure comprised 70 cycles.

Fig. 2 was obtained from the same patient on a different day, when the prevailing rhythm was again parasystole, the rate of the sinus rhythm being 75 a minute (cycle length 82). The inter-ectopic intervals measured 128 or multiples thereof. Right carotid sinus pressure again suppressed A-V conduction and, throughout the pressure, the heart action was exclusively due to idioventricular beats having the same shape in the tracing as in the preceding figure. With the exception of the first automatic beat, which differs in shape from the following ones, throughout carotid sinus pressure all were followed by ventricular extrasystoles of constant shape and with fairly accurate coupling, yielding 12 bigeminal groups. As in the preceding figure, the ectopic rhythm slowed during carotid sinus pressure. As the length of the coupling of the extrasystoles remained fairly constant, this lengthening was mainly due to a lengthening of the postextrasystolic intervals which increased from an initial 120 to a maximum of 136 , subsequently falling to 132 . The final deflections of some of the idioventricular beats suggest retrograde conduction to the atria (the 6th, 7 th, and 12 th, possibly also the 9 th and 10th beats). 


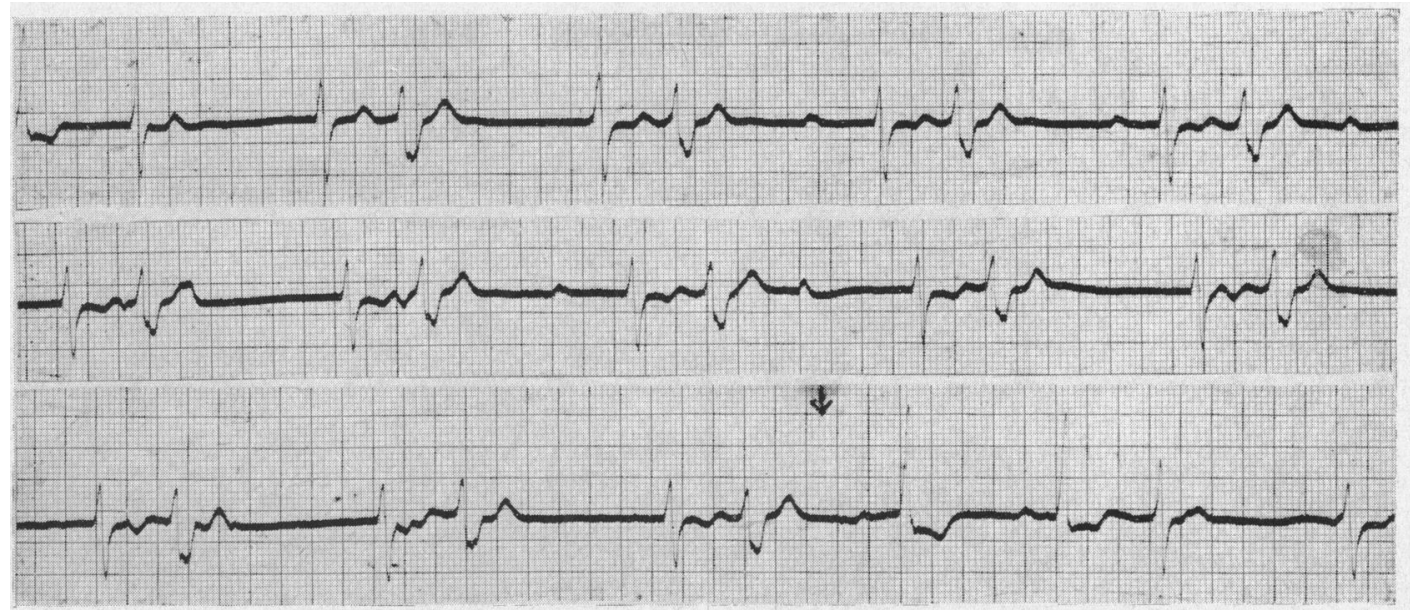

FIG. 2.-Case 1, lead II. The three strips are continuous. The tracing starts immediately after the beginning of right carotid sinus pressure; release of pressure at arrow in bottom strip. Bigeminal rhythm due to ventricular extrasystoles with accurate coupling. For further explanation, see text.

Sometimes the coupled extrasystoles varied in shape, though they had constant coupling. Fig. 3 shows 8 bigeminal groups, in which the initiating automatic beats as well as the extrasystoles varied in shape. The shape of the extrasystoles was not determined by that of the preceding automatic beat. Except for the first one, the coupling was constant at 44 . Such observations were made repeatedly.

At other times this patient had atrial fibrillation. Coupled extrasystoles occurred only after idioventricular automatic beats, never after conducted ones. Right carotid pressure then had no consistent effect on the heart rate of rhythm. The main observations in this case can be summarized as follows.

The dominant rhythm was at times sinus rhythm with ventricular parasystole. Pressure on the right carotid sinus suppressed atrioventricular conduction, the parasystolic ventricular centre being alone active at such times. During carotid sinus pressure the rate of the ectopic rhythm gradually slowed and subsequently slightly accelerated without attaining the initial rate. On several occasions the idioventricular beats were followed by ventricular extrasystoles with accurate coupling, sometimes of constant, at other times of varying shape. Coupled beats with accurate coupling were observed after automatic beats of two different shapes. These coupled extrasystoles were only-and repeatedly-observed after automatic beats during carotid sinus pressure; they never occurred after conducted beats, nor after ventricular ectopic, or fusion, beats during parasystole. At other times this patient had atrial fibrillation. Coupled extrasystoles were then also observed only after idioventricular automatic beats. Carotid sinus pressure had no consistent effect on heart rate or rhythm when the dominant rhythm was atrial fibrillation.

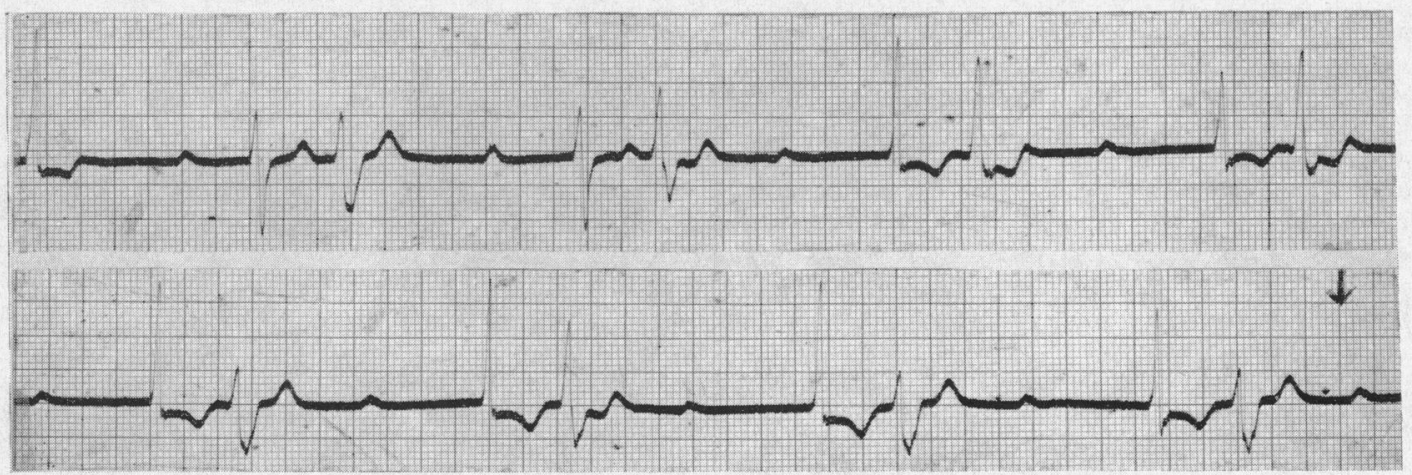

FIG. 3. Case 1, lead II. The two strips are continuous. The tracing starts immediately after the beginning of right carotid sinus pressure, the first beat in the top strip being the ventricular complex of the last conducted beat. Release of pressure at arrow in bottom strip. Bigeminal rhythm due to ventricular extrasystoles with accurate coupling. Note varying shape of automatic beats and of extrasystoles. 
Case 2. A 67-year-old woman with mitral stenosis and congestive heart failure. There was gross cardiac enlargement radiologically. The blood pressure was about 180/105. She was on digitalis and mersalyl.

Fig. 4 shows two continuous strips of lead V2. The figures immediately below the tracing refer to the lengths of consecutive cycles; those in the row below, marked P, indicate the intervals between ectopic beats of different shape and are expressed as multiples of 127-128; those in the bottom row below the bottom tracing, the coupling of extrasystoles. The reason for expressing these various intervals in this way will become apparent in the subsequent discussion. It is seen that the underlying rhythm is atrial fibrillation. The ventricular complexes are of four different shapes: the great majority are conducted supraventricular impulses, characterized by small $\mathrm{r}$, deep $\mathrm{S}$, and peaked upright $\mathrm{T}$ waves. The initial deflections measure 0.09 second. The second and fourth complexes in the bottom strip have an entirely different shape: a grossly notched bifid initial ventricular complex, measuring $0.13 \mathrm{sec}$, is followed by a sharply inverted $\mathrm{T}$ wave. These two beats, which have to be considered idioventricular automatic ones, and only these, are

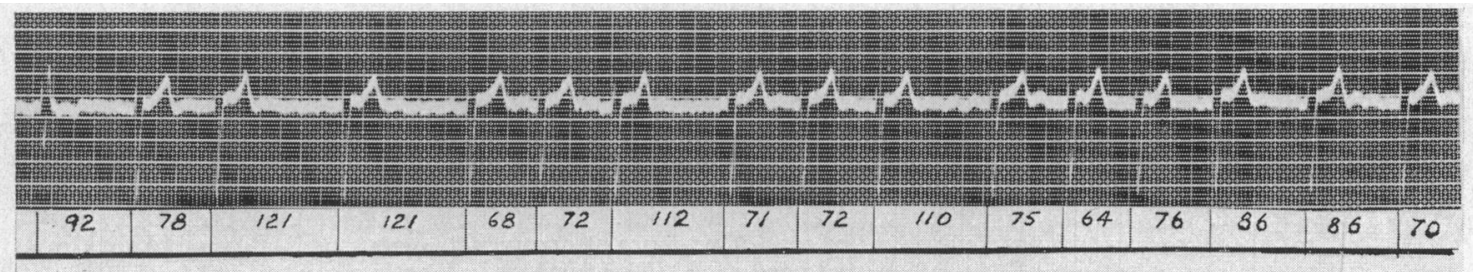

P]

$1912=15 \times 127.5$

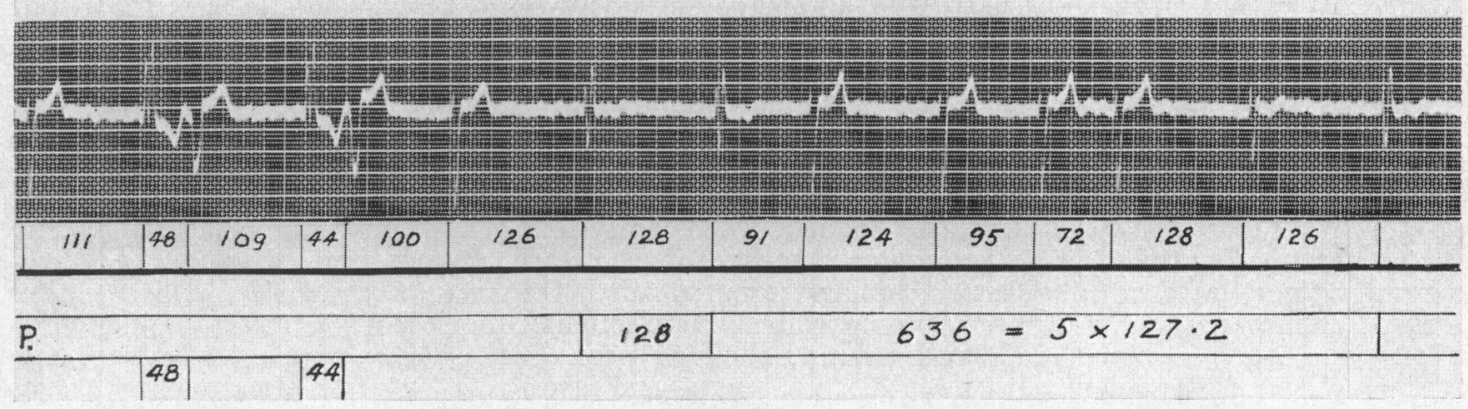

FIG. 4.-Case 2, lead V-2. The two strips are continuous. For further explanation, see text.

followed by ventricular extrasystoles with the fairly constant coupling of 48 and 44 ; their initial ventricular complexes are directed downward, followed by high upright $T$ waves. The fourth type of ventricular complexes is represented by the first beat in the top strip; and the 7th, 8th, and last complexes of the bottom strip. Their initial deflections consist of $\mathrm{rSR}^{\prime}$ complexes followed by shallow inverted T waves (differing in shape in the several beats owing to superimposition of $f$ waves). Two such complexes follow in succession in the bottom strip at an ectopic cycle length of 128, and the intervals between the other complexes of this type are all multiples of 127-128, highly suggestive of a parasystolic mechanism.

Fig. 5 shows essentially the same features. The conducted beats have higher $\mathrm{R}$ and less deep $\mathrm{S}$ waves than in lead V2. The idioventricular automatic beats have very small q, high $\mathbf{R}$, and distinct $\mathbf{S}$ waves, followed by sharply inverted $\mathrm{T}$ waves; of these, three were recorded in the top strip, all followed by ventricular extrasystoles with the same coupling $(46-48)$ as in lead V2, and having deep QS and very high peaked $\mathrm{T}$ waves; one idioventricular automatic beat recorded in the bottom strip was not followed by an extrasystole. The fourth type of complexes is represented by the third and fourth beats in the bottom strip. In this lead, they show high and grossly notched $R$ and deep $S$ waves, followed by upright $T$ waves which differ in shape and height from those of the conducted beats. It is significant that the ectopic cycle length in this lead is again 128. One noteworthy feature of this lead is that on one occasion, and on this only, a conducted impulse was followed by an extrasystole with the same coupling and of the same shape as those otherwise following only automatic idioventricular beats: this is seen at the beginning of the top strip where this extrasystole follows the second-conducted-beat. Similar observations were made in a long record obtained one week later. No extrasystole followed a conducted beat in that record.

Salient Features. The dominant rhythm was digitalized atrial fibrillation. Automatic idioventricular beats occurred in fair numbers and, with one exception, only these were followed by extrasystoles with 


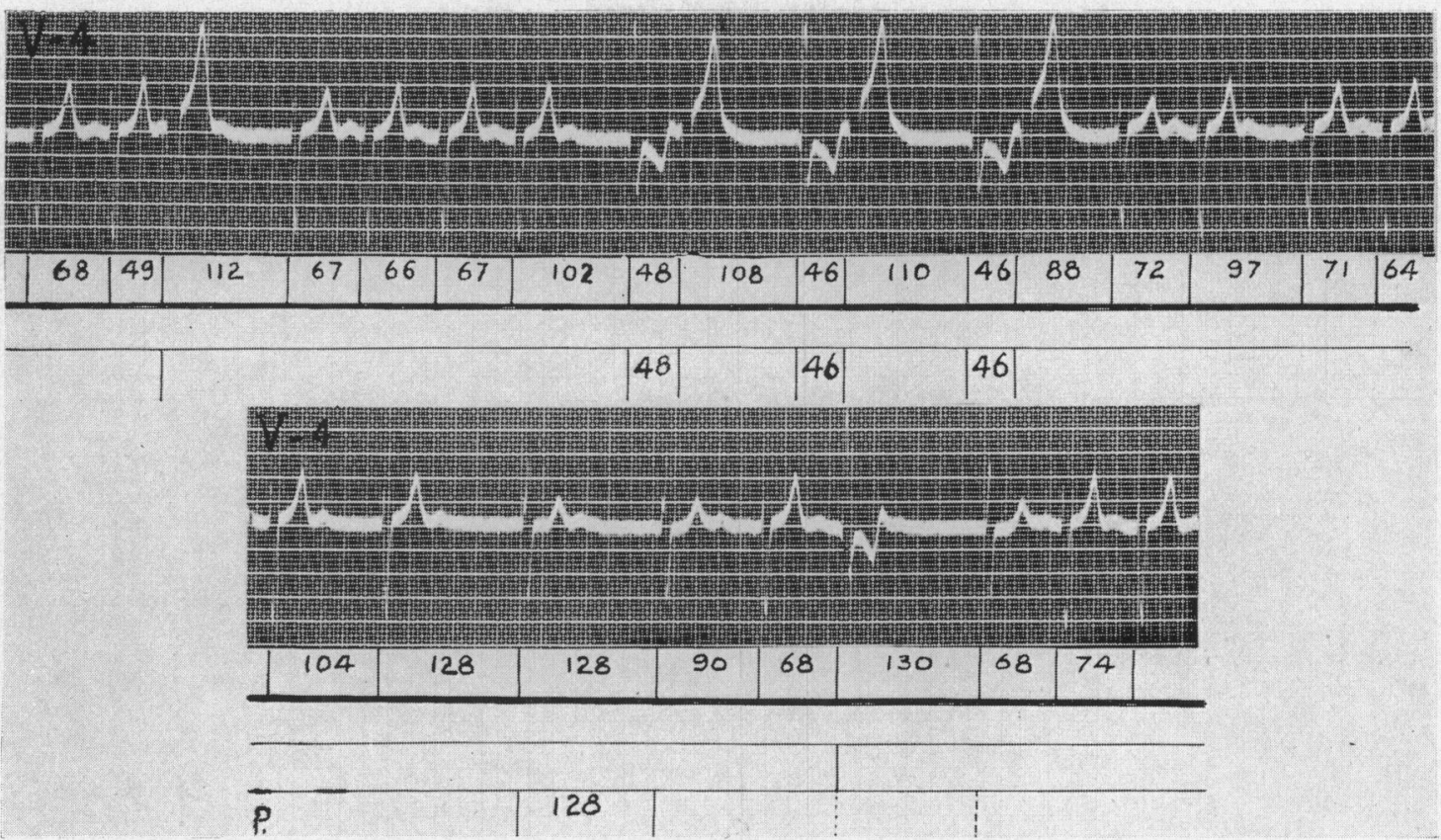

FIG. 5.-Case 2, lead V4. The two strips are continuous. For further explanation, see text.

accurate coupling and almost constant shape. In addition, a further variety of automatic ventricular beats was recorded, the ectopic cycle length of which could be directly measured as 128 ; all interectopic intervals between beats of this variety were fairly accurate multiples of 128 so that a parasystolic mechanism was established for this variety of automatic beats that was never followed by coupled extrasystoles. No parasystolic mechanism was found in respect of the former variety, namely that sometimes followed by accurately coupled extrasystoles.

Case 3. A 57-year-old woman suffering from weakness and giddiness. One year before admission she had a slight stroke from which she had made a good recovery. She was found to have left ventricular enlargement and dilatation of the aorta. Blood pressure 185/85. On admission she had visual disturbances attributed to digitalis intoxication; there was a digitalis effect also in the cardiogram. During her stay in hospital she did not receive digitalis, but was still under the influence of this drug.

The dominant rhythm was atrial fibrillation. Idioventricular automatic beats occurred fairly frequently, and both these and conducted supraventricular impulses were at times followed by extrasystoles with accurate coupling of 56. The noteworthy feature of this case is that, whenever the beginning of a period of bigeminal rhythm due to coupled extrasystoles was recorded, the extrasystole of the first bigeminal group was never initiated by a conducted beat, but invariably by an automatic one.

Fig. 6 reproduces a representative portion of lead II. It shows in its first portion four conducted beats, the interval between the first two being 208. In order to save space, the following eight conducted beats were omitted. The following portion shows three conducted beats, the last of which was followed after an interval of 208 by an automatic idioventricular beat, in turn followed by an extrasystole with a coupling of 56 . Subsequently nine bigeminal groups were recorded, of which only the last was initiated by an automatic beat. After another three conducted beats an automatic beat occurred after an interval of 212, followed by an extrasystole, again with a coupling of 56. (The distortion in the record of the extrasystole was due to a sudden technical fault of the instrument, irrevelant in the present context.) A superficial inspection suggests that only automatic beats occurring after a long interval were followed by extrasystoles, but measurement shows that this is not the case. Thus, in lead AVF (Fig. 7), a conducted beat terminating an interval of 208, and two such beats terminating intervals of 196 were not followed by extrasystoles, whereas two automatic beats occurring after intervals of 196 (not shown) and 192 were. This lead also provides another instance of the observation that once a bigeminal group had occurred that was initiated by an automatic beat, extrasystoles with the same coupling subsequently occurred after conducted supraventricular impulses. (Regarding the distortion of the extrasystoles by artefact, see above.)

The relevant points of this observation, therefore, are that, in a digitalized patient with atrial fibrillation, idioventricular automatic beats and extrasystoles with accurate coupling occurred. As distinct from the 
a

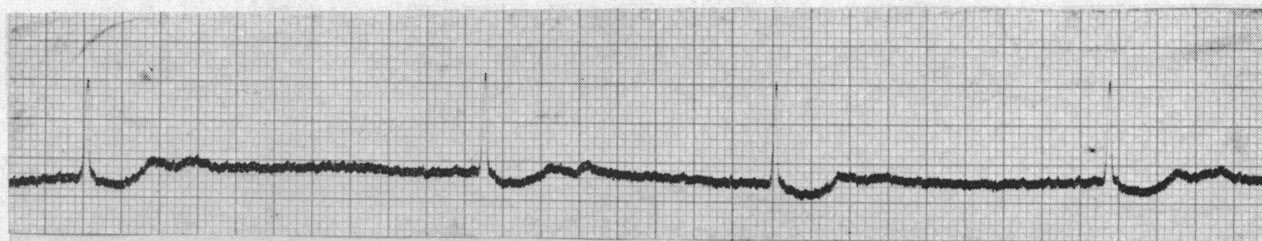

b
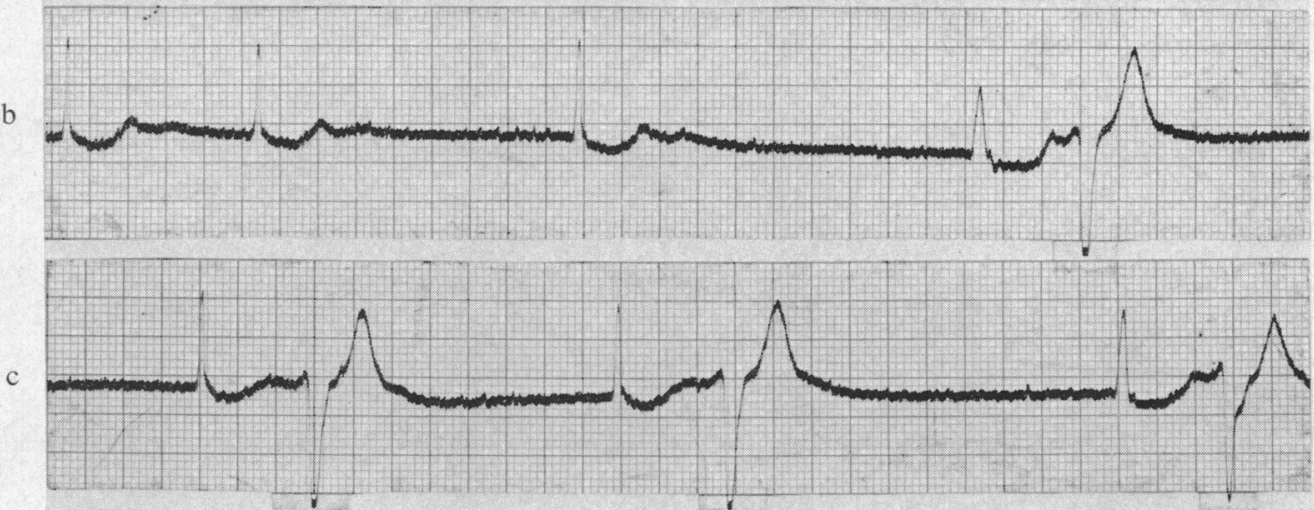

d
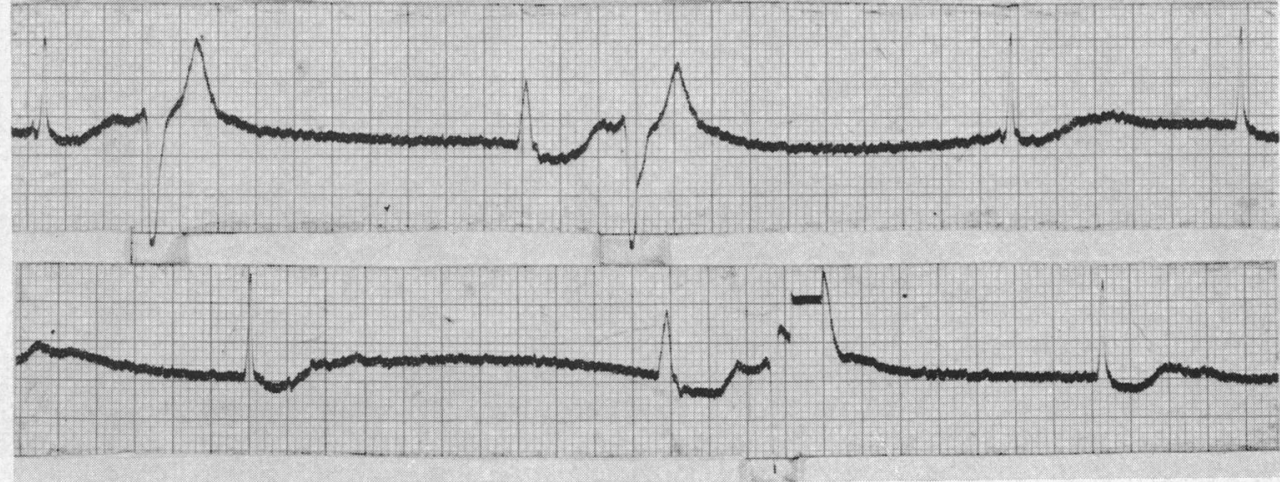

Fig. 6.-Case 3, lead II. Between a and b, eight conducted beats are omitted. b and c are continuous. Between $\mathrm{c}$ and $\mathrm{d}$, four bigeminal groups initiated by conducted beats are omitted. $\mathrm{d}$ and $e$ are continuous.

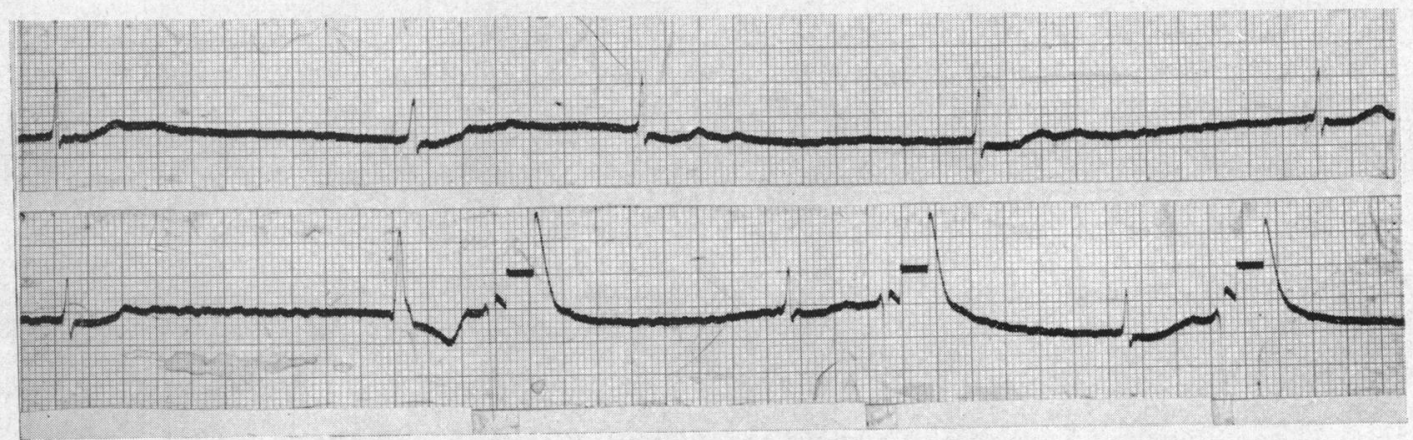

FIG. 7.-Case 3, lead AVF. Between the two strips nine beats are omitted. For further explanation, see text. 
preceding case, and even more from Case 1 and the three cases previously reported, extrasystoles frequently occurred after conducted as well as after automatic beats, but, whenever the beginning of an ectopic arrhythmia was recorded, extrasystolic bigeminal groups were always initiated by an automatic and never by a conducted beat.

\section{Discussion}

The present observations have the same feature as the three previously published cases in that automatic ventricular beats were followed by ventricular extrasystoles with fixed coupling. In some respects, however, the present observations differ from the previous ones.

In Case 1, during periods of atrial fibrillation, coupled extrasystoles were precipitated only by automatic beats. During periods of sinus rhythm and ventricular parasystole, no extrasystoles were recorded in numerous long strips; only during carotid sinus pressure, which suppressed A-V conduction so that there was only an automatic ventricular rhythm, were accurately coupled extrasystoles recorded in this patient. This is noteworthy as both the idioventricular beats of parasystole and those dominating the heart rhythm during carotid sinus pressure have to be regarded as automatic beats, originating, moreover, in the same centre because of their identity of shape in the tracings. A tentative explanation is given below. It should also be noted that, in this respect, the present observation differs from Case 3 of our previous series, in which the rhythm was atrial fibrillation and ventricular parasystole, some of the parasystolic beats, and only these, being followed by coupled extrasystoles.

In Case 2 and 3, both having atrial fibrillation like those of the previous series, not only automatic, but also some conducted beats were followed by extrasystoles. In Case 2 this was observed only after one supraventricular beat; in Case 3, extrasystoles occurred after many, but only after the bigeminy had been initiated by an automatic beat.

All patients, including the three described in the previous communication, had advanced structural heart disease and all were under the influence of digitalis.

The present three observations, as well as the three previously reported, are special instances of one ectopic focus being awakened-exclusively or preferentially-by another ectopic focus. In various forms this phenomenon has been observed both experimentally and clinically: experimentally, in connection with ectopic ventricular tachycardia elicited by the topical application of aconitine (Scherf et al., 1950; Scherf and Schott, 1953, p. 232 and Fig. 149); clinically, in connection with ectopic ventricular rhythms following myocardial infarction (Scherf and Schott, 1953, Fig. 27); with the precipitation of atrioventricular tachycardia by ventricular extrasystoles (Castellanos et al., 1955); and with that of upper A-V nodal beats by ventricular extrasystoles with retrograde conduction (Schott, 1955). The main difficulty is, however, to understand why the ectopic focus was activated only, or preferentially, by another ectopic beat, and not, or not constantly, by supraventricular conducted beats.

The possibility of return extrasystoles has to be considered and this mechanism cannot be excluded for extrasystoles following those automatic ectopic beats whose shape suggests retrograde conduction (Case 1, Fig. 2, some beats, see text above, possibly also some beats in Fig. 3). However, this explanation can certainly not hold good in cases of atrial fibrillation, and is unlikely in those instances in which the precipitating automatic beats had no features pointing to retrograde conduction, particularly if beats containing such features were available for comparison, as in Case 1 .

In our previous paper we offered as a tentative explanation the assumption that a directional element was present, refractory tissue in the vicinity of the centre or centres of extrasystolic impulse formation causing a protective block of such centre(s) against the supraventricular impulses, and giving access to the initiating beat only from certain directions. We believe that such an explanation, though again tentative, is still the most satisfactory for the whole series.

In support of our view we quoted observations on unidirectional block, postulated to account, for instance, for the retrograde conduction of idioventricular beats to the atria in patients with complete atrioventricular block, which is not uncommon. The word "block" should be qualified. 
As we pointed out elsewhere in a somewhat different but related context (Scherf and Schott, 1953, pp. 173, 518), the older concept of a wall of refractory tissue spherically surrounding an ectopic centre is unsatisfactory as a general explanation of a "protective block" of such a centre. It certainly could not account for the phenomenon under discussion, for, if an impulse cannot reach an ectopic centre from one direction, it should do so from another direction within a few thousandths of a second. Nor would it account for the observation that impulses of one kind of ventricular beats activate such a centre whereas those of another kind do not have this effect. In our view, the "block" of such centre is related to the ratio strength of impulse: excitability. Some more recent reports seem relevant in this context. Thus, Rubio and Rosenblueth (1956) found in dogs considerable differences in velocity of conduction between the two ventricles, depending on the direction of excitation (right-to-left compared with left-to-right ventricle), which these authors attributed to the type of polarization of the interventricular septum. A different manifestation of a directional element was found by Rosenblueth (1955) in experiments on dogs in which a slow A-V rhythm was produced. Two impulses sent through the A-V node in a V-A direction were followed by automatic nodal A-V beats, but not when sent at the same interval in the opposite direction. The same group of workers found directional elements to be of importance in nerves (Rosenblueth et al., 1949). Applying these observations to the heart, Rosenblueth and Rubio (1955) stressed the importance of the relation between threshold of excitability in various parts of the heart and amplitude of action potential, in the causation of different velocities of conduction in different directions.

These observations support our conception of the nature of "block" of an ectopic centre, and provide a basis for the assumption that, in certain circumstances, a directional element may be introduced by the site of refractory tissue and its degree of refractoriness, affecting velocity, and presence or absence, of conduction.

Elsewhere, we have given reasons for our belief that extrasystoles in the strict sense of the term as used by us-that is, with accurate coupling to the preceding beat-arise in a circumscribed focus where they are initiated by the preceding beat; and that in the extrasystolic focus there are local nonpropagated potentials which, during a period of temporarily enhanced excitability following an initiating beat, become supra-threshold, giving rise to a propagated potential — the extrasystole (Scherf and Schott, 1953).

This view has been supported by more recent observations. The presumed origin of ectopic beats in a circumscribed focus with the above characteristics is in agreement with the observation, made by means of intracellular electrodes, that local potential changes can be demonstrated in spontaneously beating Purkinje tissue (Weidmann, 1951; Trautwein et al., 1953). That during the phase of enhanced excitability consequent upon an initiating beat, namely, during its after-potential associated with supernormality, such local non-propagated potentials may become suprathreshold and give rise to a propagated impulse was first demonstrated in the heart of the snail by Arvanitaki (1938), and by Bozler (1943) in the heart of the turtle. In a study on large numbers of ventricular extrasystoles in man, Lepeschkin and Rosenbaum (1957) have established that the coupling of the extrasystoles is closely related to the inscription of the $U$ wave, which is an expression of the negative after-potential. This observation not only supports our views about the origin of extrasystoles, but also explains certain phenomena about their incidence. Thus, Langendorf et al. (1955) have found that, in atrial fibrillation, extrasystoles with fixed coupling tend to occur after long ventricular cycles. This can be attributed to the fact that a slow heart rate tends to elevate the $U$ wave, indicative of an increased excitability after longer ventricular cycles (Lepeschkin and Rosenbaum, 1957).

Regarding the site of the focus of origin of the extrasystole, as one of two possibilities we suggested (Scherf and Schott, 1953, p. 517) that such a focus may be situated in an area beyond a "block" and that the temporarily increased excitability is akin to Wedensky facilitation.

Wedensky (1903) found in nerves that the threshold of the segment below a block, induced in various ways such as cold, drugs, or pressure, becomes lower than it was before; this phenomenon was studied in more detail with modern methods by Hodgkin (1937) and Lorente de Nó (1939).

If our assumption is correct that, in the type of case reported in the present and previous com- 
munications, there is a directional element regarding the initiating beat, this would depend on the site of refractory tissue in the vicinity of the extrasystolic centre(s). The assumption of the presence of areas of depressed conduction (refractory tissue) is made probable by the fact that all patients of these series had advanced structural heart disease. The part played by digitalis in causing the phenomenon under discussion is difficult to evaluate, in view of the complex effect of the drug on the refractory period. It seems to us quite probable, however, that it is more than a coincidence that all patients were under the influence of this drug, which is known to increase the refractory period of the conducting system on the one hand, and to promote ectopic impulse formation on the other. Since the length of the refractory period is also known to be largely influenced by functional changes, for example the ionic milieu and $p \mathrm{H}$, the area of depressed conduction need not be assumed to be a fixed entity. Functional changes in extent and degree of refractoriness seem most probable. This would make it understandable that, in some cases, only impulses from certain directions, namely, automatic beats, precipitate coupled extrasystoles, whereas, in others, extrasystoles may also be elicited by supraventricular beats, but occur preferentially after automatic ones.

In our previous paper we discussed the alternative possibility that the idioventricular automatic and the extrasystolic beats originate in the same centre, the different shape of the extrasystole being due to aberrant ventricular conduction. We pointed out that, for stated reasons, we believed the origin of the extrasystole in a focus distant from that of the idioventricular beat to be far more likely. The same arguments hold good when the whole series of six cases is considered.

The observation made in Case 1, that coupled extrasystoles occurred only during idioventricular rhythm prevailing during carotid sinus pressure, but not after idioventricular parasystolic beats of the same shape outside carotid sinus pressure, requires an explanation. We believe that this is due to the excitatory effect of acetylcholine due to vagal stimulation, which is one important effect of carotid sinus pressure. This aspect of the action of acetylcholine and vagal stimulation has attracted a great deal of attention recently, largely due to the work of Burn and his collaborators (Burn, 1950; Burn et al., 1955; Burn and Rand, 1957). Marshall and Williams (1956) studying, in isolated rabbit atria, pacemaker potentials and the excitation by acetylcholine found that, at certain low temperatures, only non-propagated pacemaker potentials were recorded, and ACh then caused propagated action potentials and auricular contraction. ACh was thus shown in certain circumstances to restore a link between a pacemaker and the surrounding tissue. As early as 1923, Lewis and Drury found in dogs that vagal stimulation abolished intraauricular block produced by clamping or cooling, and that acetylcholine had the same effect. Marshall and Williams also stated that "... the slowly rising foot preceding the action potential would be seen in any region which was firing spontaneously, and that the production of such 'pacemaker' potentials is not the prerogative of a specific anatomical region of the heart". (See also Trautwein and Gottstein, 1953.) It seems reasonable, therefore, to apply these considerations also to ectopic foci.

Furthermore, digitalis is known to potentiate the effect of acetylcholine or of vagal stimulation on the heart (Perry and Reinert, 1954 (with references to earlier publications); and McEwen, 1956).

This view accords well with another observation made in this patient, namely, that the rate of the idioventricular rhythm during carotid sinus pressure gradually slowed. This effect, on the ectopic rhythm, of carotid sinus pressure in cases of ventricular parasystole has been observed before in patients under the influence of digitalis (Golbey et al., 1954; Scherf et al., 1957), release of acetylcholine being considered as the likely cause.

A further possible factor favouring the occurrence of coupled extrasystoles only during idioventricular rhythm resulting from carotid sinus pressure may be the longer cycle lengths of the idioventricular rhythm as compared with the coupling of the ectopic beats during sinus rhythm and parasystole. As mentioned above, a slow heart action tends to increase the height of $U$ waves, associated with increased excitability, and thus facilitates the occurrence of extrasystoles. The shape of some of the automatic beats that precipitated extrasystoles is suggestive of this possibility. 


\section{SUMMARY}

Observations are described on three patients with ectopic ventricular arrhythmias in whom coupled extrasystoles were precipitated, either exclusively or preferentially, by automatic idioventricular beats, but followed conducted supraventricular beats either not at all or only on one single occasion or only when bigeminy had been initiated by an automatic beat. In one patient, coupled extrasystoles were precipitated only by idioventricular beats, when they alone activated the heart during carotid sinus pressure, but neither by ventricular automatic beats during sinus rhythm with parasystole nor by conducted supraventricular beats during atrial fibrillation. This phenomenon, and also the observed slowing of the idioventricular rhythm during carotid sinus pressure, are attributed to the excitatory effect of acetylcholine, and possibly also to the lower heart rate prevailing at such times. In the two other patients, the dominant rhythm was atrial fibrillation. These observations are considered in conjunction with those previously reported by us on three patients with closely related arrhythmias. All patients had advanced structural heart disease and were under the influence of digitalis. The assumption of a directional protective "block" of the extrasystolic centre, due to the presence of refractory tissue in the vicinity of the centre, is again put forward as the most satisfactory, though still tentative explanation. By its location, and degree of refractoriness, at a given time, such refractory tissue would allow the initiating impulse to have access to the extrasystolic centre from certain directions, but not from others. It is emphasized that such "block" should not be visualized as a spherical wall of refractory tissue surrounding the centre, but as being related to the ratio strength of impulse: excitability. Functional changes in the site, extent and degree of refractoriness, which are greatly influenced by the ionic milieu and $p \mathrm{H}$, among other factors, are a likely cause for the observation that, in some cases, coupled extrasystoles follow only automatic beats, whereas, in others, extrasystoles may also be precipitated by supraventricular beats, but occur preferentially after automatic ones. The origin of an extrasystole is assumed to be due to local, non-propagated pacemaker potentials in the centre, temporarily becoming suprathreshold during a period of enhanced excitability consequent upon the initiating beat, and the underlying mechanism is considered as being akin to Wedensky facilitation in nerve.

\section{REFERENCES}

Arvanitaki, A. (1938). Propriétés rythmiques de la matière vivante. Actualités scientifiques et industrielles, 761 and 762. Hermann, Paris.

Bozler, E. (1943). Amer. J. Physiol., 138, 273.

Burn, J. H. (1950). Proc. roy. Soc. B., 137, 281.

-, and Rand, M. J. (1957). J. Physiol., Lond., 138, 172.

—, Vaughan Williams, E. M., and Walker, J. M. (1955). J. Physiol., Lond., 128, 277.

Castellanos, A., Cano, L. A., Calviño, J. M., and Taquechel, N. (1955). Rev. cuban. Cardiol., $16,419$.

Golbey, M., Ladopulos, C. P., Roth, F. H., and Scherf, D. (1954). Circulation, 10, 735.

Hodgkin, A. L. (1937). J. Physiol., Lond., 90, 183, 211.

Langendorf, R., Pick, A., and Winternitz, M. (1955). Circulation, 11, 422.

Lepeschkin, E., and Rosenbaum, M. B. (1957). Circulation, 15, 82.

Lewis, T., and Drury, A. N. (1923). Heart, 10, 179.

Lorente de Nó, R. (1939). J. Neurophysiol., 2, 402.

McEwen, L. M. (1956). J. Physiol., Lond., 131, 678.

Marshall, J. M., and Vaughan Williams, E. M. (1956). J. Physiol., Lond., 131, 186.

Perry, W. L. M., and Reinert, H. (1954). Brit. J. Pharmacol. Chemotherap., 9, 324.

Rosenblueth, A. (1955). Arch. Institut. Cardiol. Mexico, 25, 171.

-, Alanis, J., and Mandoki, J. (1949). J. cell. comp. Physiol., 33, 405.

- and Rubio, R. (1955). Arch. Institut. Cardiol. Mexico, 25, 535.

Rubio, R., and Rosenblueth, A. (1956). Arch. Institut. Cardiol. Mexico., 26, 644.

Scherf, D., Morgenbesser, L. J., Nightingale, E. J., and Schaeffeler, K. T. (1950). Cardiologia, Basel, $16,232$.

- , and Schott, A. (1951). Amer. Heart J., 41, 291.

- _ (1953). Extrasystoles and Allied Arrhythmias. W. Heinemann, London, and Grune \& Stratton, New York.

, Reid, E. C., and Chamsai, D. G. (1957). Cardiologia, Basel, 30, 217.

Schott, A. (1955). Brit. Heart J., 17, 247.

Trautwein, W., and Gottstein, U. (1953). Naturwissenschaften, 40, 443.

- , - and Federschmidt, K. (1953). Pflüg. Arch. ges. Physiol., 258, 243.

Wedensky, N. E. (1903). Pflüg. Arch. ges. Physiol., 100, 1.

Weidmann, S. (1951). J. Physiol., Lond., 115, 227. 